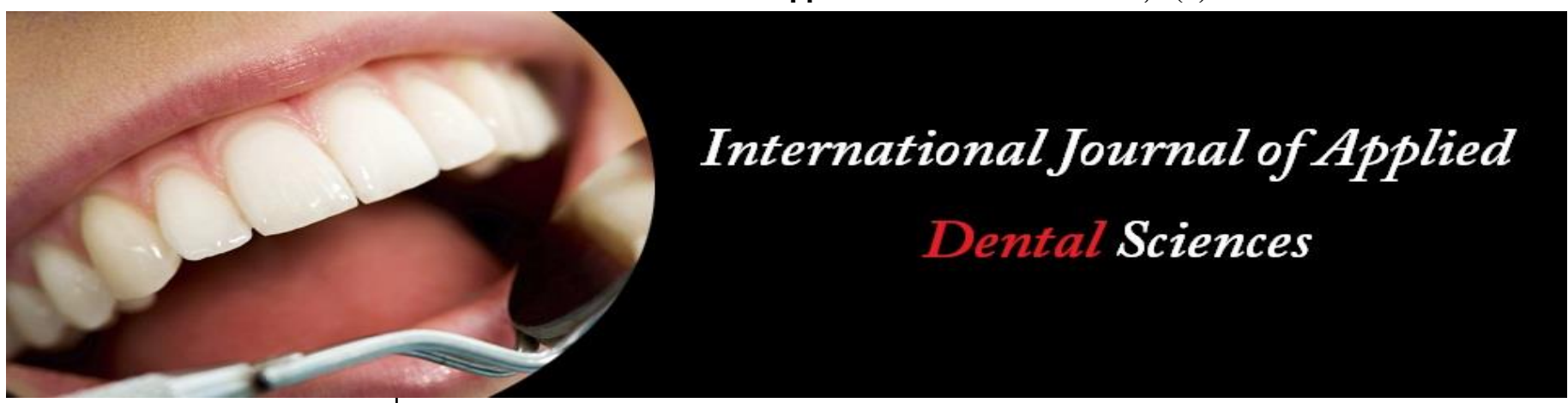

ISSN Print: 2394-7489

ISSN Online: 2394-7497

IJADS 2020; 6(3): 601-609

(C) 2020 IJADS

www.oraljournal.com

Received: 15-05-2020

Accepted: 06-07-2020

Dr. Muhammad Ziad Sultan

Professor, Department of

Paediatric Dentistry, Faculty of

Dentistry, University of Hama,

Syria

Moulham ALhasan

Postgraduate Student,

Department of Paediatric

Dentistry, Faculty of Dentistry,

University of Hama, Syria
Corresponding Author:

Dr. Muhammad Ziad Sultan

Professor, Department of

Paediatric Dentistry, Faculty of

Dentistry, University of Hama,

Syria

\section{In vitro study to evaluate the effect of $2 \%$ chlorhexidine solution application on composite restorative bonding strength of primary teeth dentine}

\section{Dr. Muhammad Ziad Sultan and Moulham ALhasan}

DOI: https://doi.org/10.22271/oral.2020.v6.i3i.1014

Abstract

Introduction: With the increasing use of composite resin as a cosmetic restorative material at the level of posterior teeth, the trend of clinical and laboratory studies to research mechanical and physical properties related to composites and modern linking systems accompanying their use; The aim is to improve the results of the use of each of them for the better.

Aim of the Study: To assess the effect of applying chlorhexidine solution $2 \%$ on the forces of bonding compost with temporary dentine, by assessing the marginal leakage.

Materials and Methods: The study included 40 healthy, newly extracted, temporary human molar, distributed equally and randomly to two groups, group (1) restored with composite without applying chlorhexidine solution $2 \%$, while group (2) was filled with composites with application of chlorhexidine solution $2 \%$ for 60 seconds and after acid scratching Through them, the edge leakage was studied.

The composite resin fillings are prepared and then kept it for 6 months with distilled water. The edge leakage test was done with a stereo microscope, with a $20 \%$ magnification.

Results :The application of chlorhexidine $2 \%$ solution did not adversely affect the marginal leakage.

Keywords: Temporary teeth, temporary dentine, compost, flange leakage

\section{Introduction}

Materials used in the research

Chlorhexidine solution 2\% YG - HEXIDINE 2\%) from YG - Dent in a package containing $60 \mathrm{ml}$ of the product, composed of Chlorhexidine Digluconate $2 \%$ in addition to Methylparaben (a preservative) and deionized water. This substance is a strong bactericide agent within this concentration and is used by the manufacturer to irrigate root canals and to purify pits before applying various restorative materials.

\section{Composite resin group}

- Tetric N- Etch from Ivoclar Vivadent, a phosphorous acid of 37\% concentration, knurled to the ivory port.

- Ivoclar Vivadent Tetric N-Bond is classified as a binding substance for the fifthgeneration port and ivory, photosynthetic, single-packaging.

Restored material: Ivoclar Vivadent Tetric N-Ceram resin is a Nanohybrid nanoparticle hybrid resin with blue light and wavelength (400 - 500) $\mathrm{nm}$. It is a shady material on the beam that consists of:

Dimethacrylates make up $19-20 \%$ by weight fillers that contain barium glass, etherbium trifluoride, and copolymers, and constitute 80 to $81 \%$ of the weight.

Other additions include accelerators, preservatives, and dyes, which account for less than $1 \%$ of the weight.

Chloramine $\mathrm{T}$ powder from Medex, from which a 5\% solution was prepared with a solution of $5 \mathrm{~g}$ within a liter of water. 
methelyne blue from Dako, a bluish-brown powder that gives a blue pigment when dissolving in water at a concentration of $1 \%$

Distilled water

Airtight plastic containers

Glue wax - nail polish - timer

Disc separator mounted on a slow speed grip

Glass sheets

Binding - Vaseline - Self-Propelling Acrylic - Cleaning Brushes

* All materials were handled according to the manufacturer's instructions

\section{Search devices}

1. COXO DB 685 LED optical hardener emits a blue light with a wave of 440-480 nanometers and with several systems (continuous, pulsed, graded intensity). With a beep every 5 seconds (which is the mode used for this search)

2. High-speed turbo grip with DIA-BURS heavy duty diamond and funnel bands in various sizes and straps

3. Appledental straight grip

4. Top-mounted magnifying glass works with two $20 \mathrm{X}$ and 40X magnifications

5. Thermo-cycle device (water bath) company (Emmevi)

\section{Research Sample}

The sample of the study included 40 temporary lower and upper healthy intravenous molar due to an orthodontic reason or approaching its physiological fall, as the ages of the children from whom the sample was collected ranged from 9 to 11 years old, collected from dental clinics and the Faculty of Dentistry University of Hama.

\section{Criteria for selecting the research sample}

1. That the temporary crowns of the crowns are intact and free from any necrosis or restoration.

2. The crowns should be free from defects of development, manufacture and defects of calcification.

3. To be free from any visible fracture or crack.

Preparing the sample: ISO / TS 11405: 2003 (E) Standards
Instructions were adopted for the study of adhesion to dental structures, as follows: First, the teeth were washed with running water immediately after extraction, and then the soft tissue residues adhering to the teeth were removed with a scaling tool, to be preserved after that These teeth are sealed plastic containers containing $0.5 \%$ chloramine $\mathrm{T}$ solution for a week, then the teeth were then transferred to plastic containers containing distilled water, and kept at a temperature of $4{ }^{\circ} \mathrm{C}$ in the fridge for a month, taking into account renewing the storage medium periodically every week until the sample is complete .

After completing the number of the research sample, the necessary work was done, and the sample was preserved for 6 months with distilled water.

The tooth port was then drilled using a diamond spikemounted turbine grip to reveal surface ivory with continuous hydration with water, with the aim of forming a smear layer of laboratory-standardized parameters ${ }^{[4]}$.

The ivory surface exposed to a diameter of at least $5 \mathrm{~mm}$ was smoothed using smooth-graded glass sheets (120-240-400600) moisturized and fixed on a flat and hard surface until obtaining a smooth and flat ivory surface, taking into account the immersion of the tooth with distilled water after direct use of each measurement From the above mentioned glass paper measurements; In order to maintain the moisture of ivory and prevent drying out.

\section{How it works}

\section{The work included the following stages}

1 -Study the effect of applying chlorhexidine solution $2 \%$ for a period of 60 seconds after acidic scratching and before performing compost filling on temporary dentine by studying the edge leakage.

The study was conducted by preparing a fifth class pits on the vestibular surface of the extracted teeth.

A fifth class pit was prepared in the middle of the vestibular surface of each of the sample items by a diamond spike with a cylindrical head with a length of $4 \mathrm{~mm}$ and a diameter of 1 $\mathrm{mm}$, so that the depth of the hole was half the length of the working head of the spike (i.e. $2 \mathrm{~mm}$ ) and in a way that we did not reach the pulp chamber and the diameter was equal to the diameter of the spike That is $(1 \mathrm{~mm})$.

Table 1: The edge leakage was studied in two groups

\begin{tabular}{|c|c|c|}
\hline The first group & 20 temporary molar & without applying chlorhexidine solution \\
\hline The second group & 20 temporary molar & with application of chlorhexidine solution \\
\hline
\end{tabular}

The first group: the composite was applied in the traditional way without applying chlorhexidine solution.

The composite was applied in the traditional way without applying chlorhexidine solution according to the following: Dental ivory was scratched with $37 \%$ phosphorous acid for 15 seconds (according to the manufacturer's instructions), then washed the teeth with water under air pressure for 15 seconds, then dried the surface of the ivory until seeing the chalky appearance, then applied the bonding substance and hardened it for 20 seconds, and from Then applying the composite filling within standardized molds to all the teeth of the sample so that the filling diameter ranged between $3 \mathrm{~mm}$ and a length of $5 \mathrm{~mm}$, then the process of hardening the filling using a lightning hardening device DB 685 LED for COXO produces a blue light with a wave of 440-480 nanometers, and with a continuous hardening system for a period of 40 seconds So that the head of the device is as close as possible to the resinous material and perpendicular to it, after completing the work of all the teeth of the aforementioned sample, the teeth were kept with distilled water for a period of 6 months.

The second group: the composite was applied in the traditional way in addition to applying the solution of chlorhexidine $2 \%$ for 60 seconds and after acid scratching.

Dental ivory was scratched with $37 \%$ phosphorous acid for 15 seconds (according to the manufacturer's instructions), then washed the teeth with water under air pressure for 15 seconds, then dried the ivory surface until seeing the chalky appearance, then applied the $2 \%$ chlorhexidine solution for a period of 60 seconds, Then conduct continuous aqueous washing with air pressure for 15 seconds, then dry the ivory surface and apply the bonding substance and harden it for 20 seconds, then apply the compost filling within standardized molds.

$5 \mathrm{~mm}$, then the filling process is hardened using a COXO DB 685 LED optical hardening device that produces a blue light 
with a wave of 440-480 nanometers, and with a continuous hardening system for a period of 40 seconds so that the head of the device is as close as possible From the resinous material and perpendicular to it, after completing the work of all the teeth of the aforementioned sample, the teeth were kept with distilled water for a period of 6 months.

The tooth was kept after restoration for 6 months at room temperature. With the aim of approaching the laboratory situation and the clinical situation in terms of the exposure of the restored substance to thermal changes when it is placed in the oral environment as a result of eating foods and liquids at different temperatures, 500 thermal cycles were conducted between two temperatures (5-55) degrees Celsius using the water temperature, which is the procedure followed Widely used in Sunni research ${ }^{[5]}$.

The apical foramen was then closed for each of the sample items with adhesion wax, then the surface of each tooth was painted with a first layer, then a second layer of nail polish, except for the restoration surface and $1 \mathrm{~mm}$ around its circumference then the teeth were immersed in a methylene blue solution that was prepared with a concentration of $0,5 \%$ for 24 hours, then the teeth were washed with running water. Each tooth was cut vertically towards the cervical cutter and from the middle of the filling using a separating disk with profuse aqueous cooling so that we obtained a palatal vestibular section passing from the middle of the restoration, and the surfaces were polished with a rotating diamond softening disc moisturized by Continuous water cooling; In order to obtain a flat and glossy surface that can be read under optical microscopy, the surface of the samples was then thoroughly washed with water to get rid of any residue or debris attached to it after polishing, and samples were photographed using a Stereo Micro-scope found in the Department of Life Sciences - Faculty of Medicine Dental University of Hama. The edge leakage was evaluated according to the depth of influence of the colored material as follows:

\section{Class 0 - No edge leakage}

Grade 1 - there is an edge sealant and does not exceed half of the filling

Grade 2 - There is an edge sealant and exceeds half of the filling and does not exceed the entire filling

Grade 3 - The edge leakage exceeds the ends of the filling The pictures were read by researchers in the Department of Pediatric Dentistry without knowing the identity of the materials used, and the results were recorded in the research form for the study of the severity of the edge leakage.

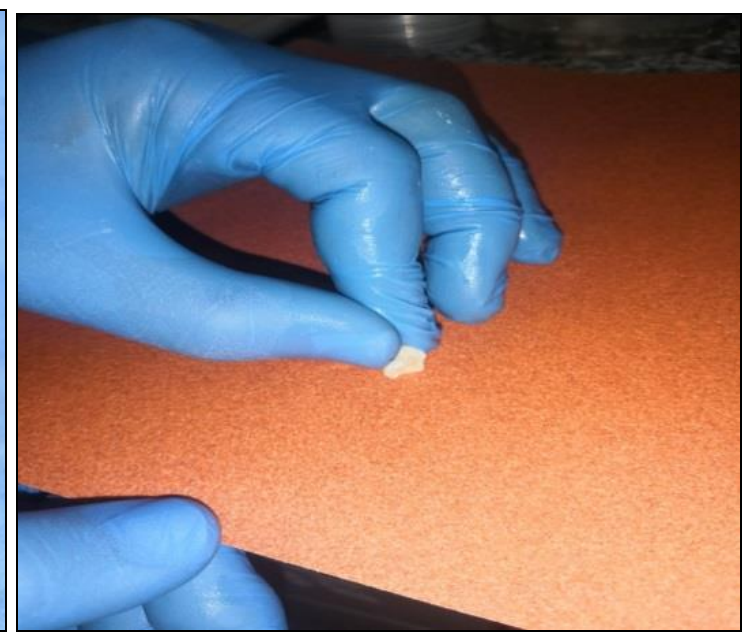

Fig 2: Cold tooth process with silicon glass paper

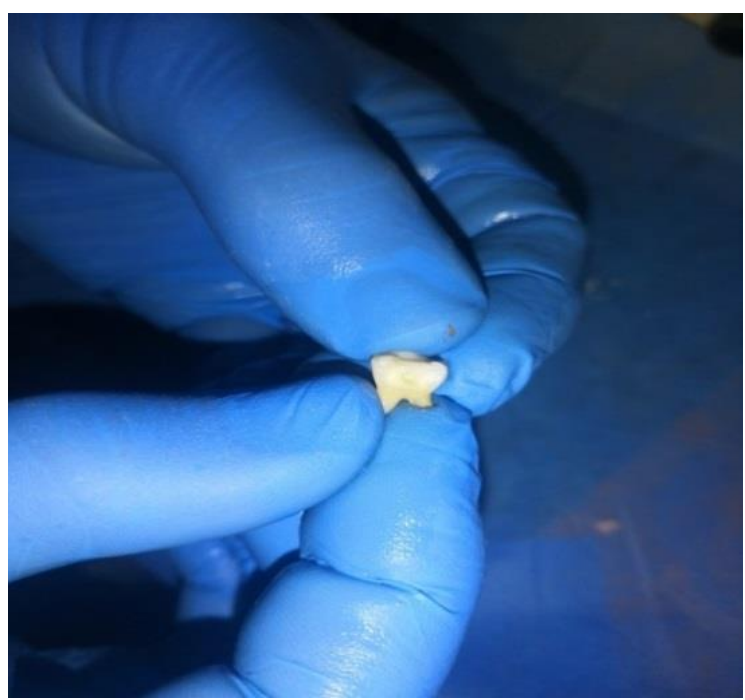

Fig 4: Acid knurling process
Fig 3: pit preparation process

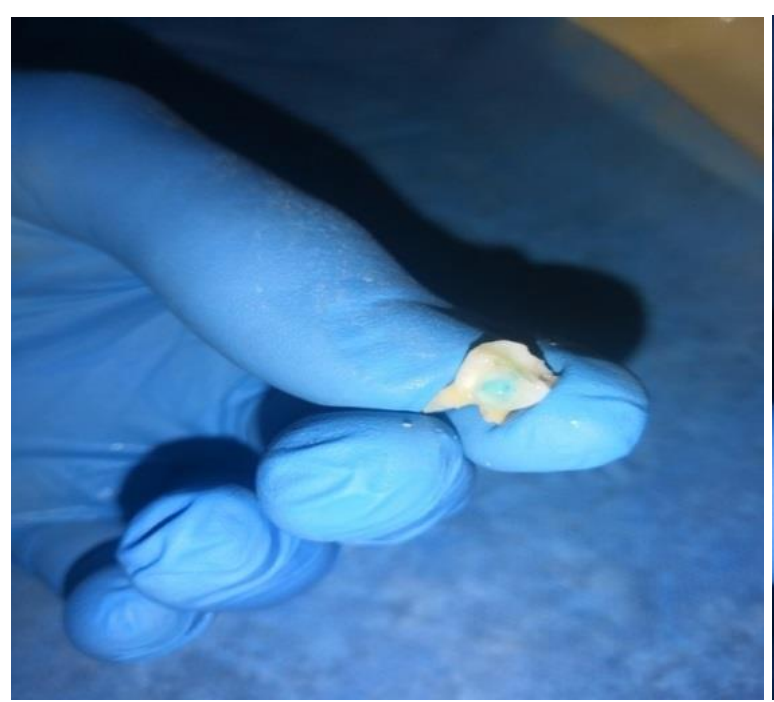

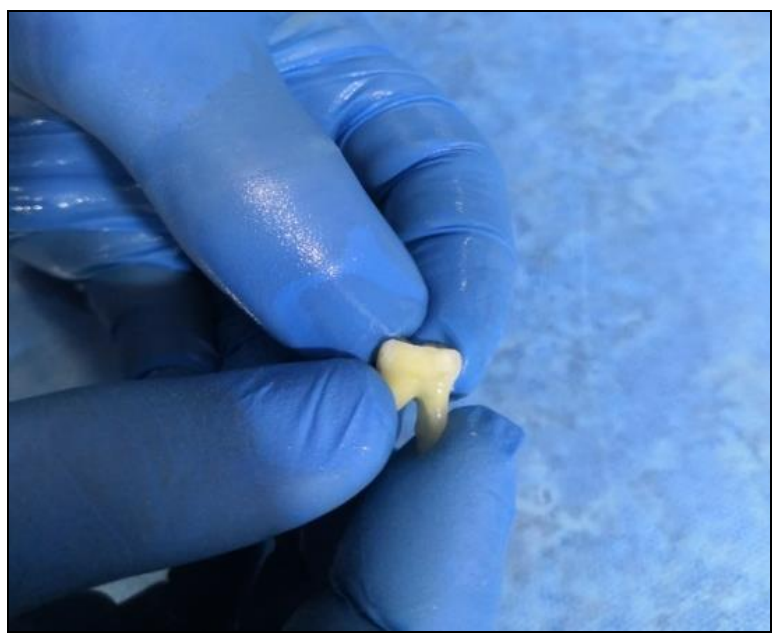

Fig 1: Cutting the port of the vestibular surface.

$2003 \sim$ 


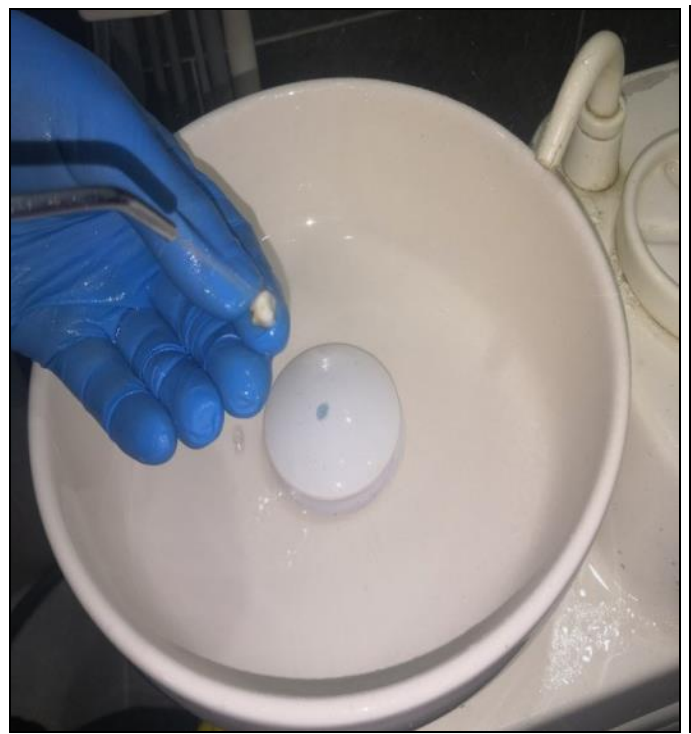

Fig 5: Washing process with running water

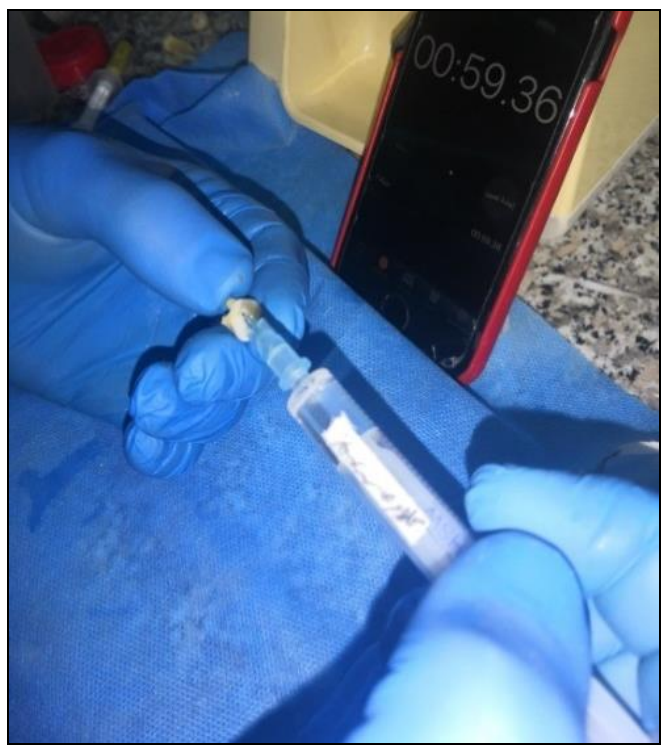

Fig 7: Application of chlorhexidine

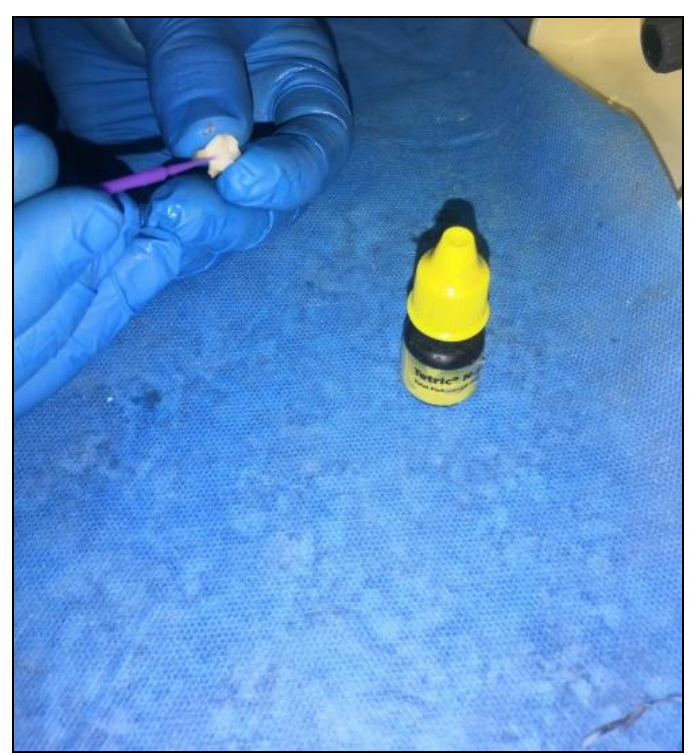

Fig 9: Application of the binding material

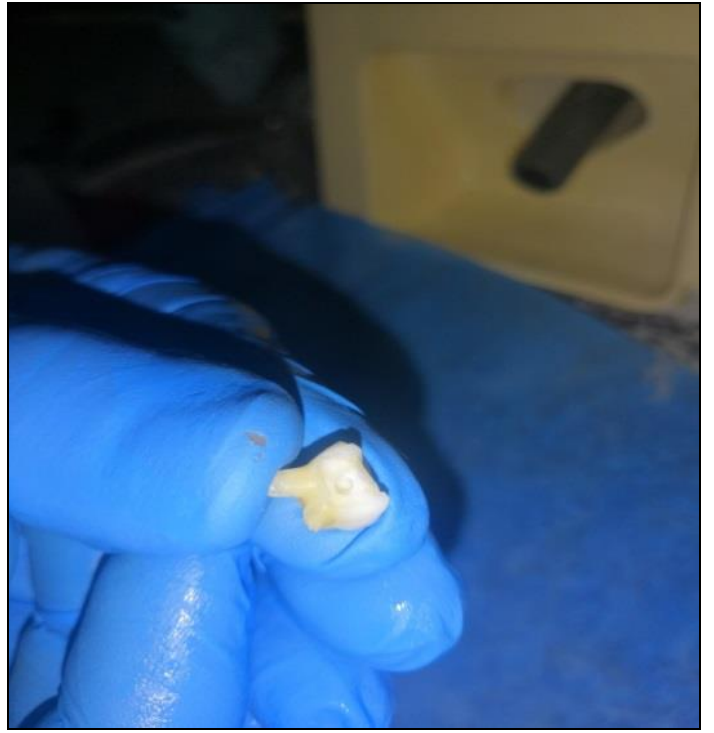

Fig 6: chalky appearance

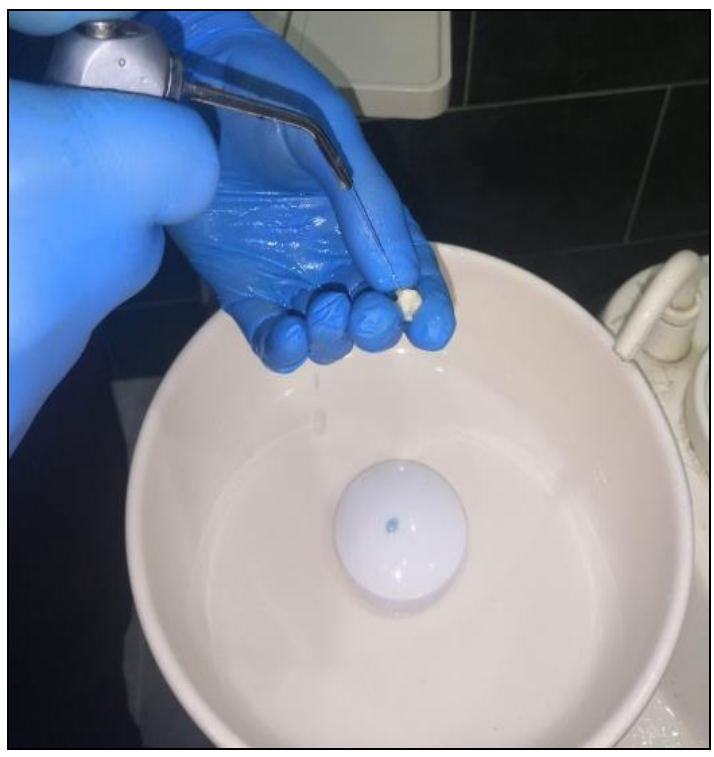

Fig 8: Washing with running water

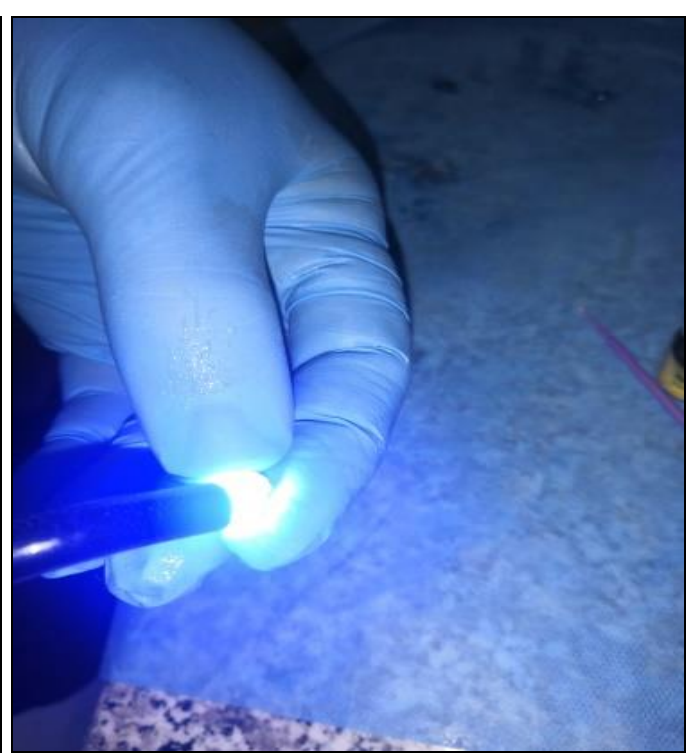

Fig 10: Solidification of the binding material 


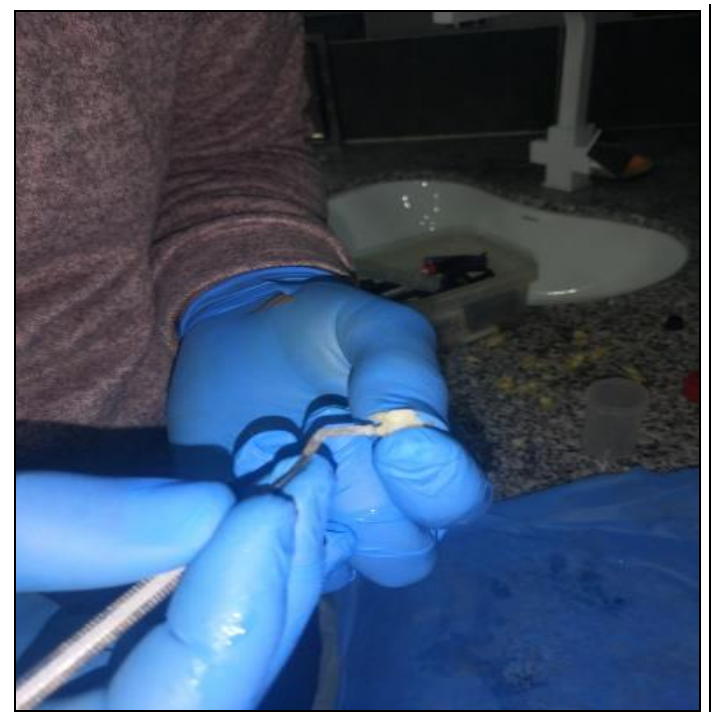

Fig 11: Application of the composite.

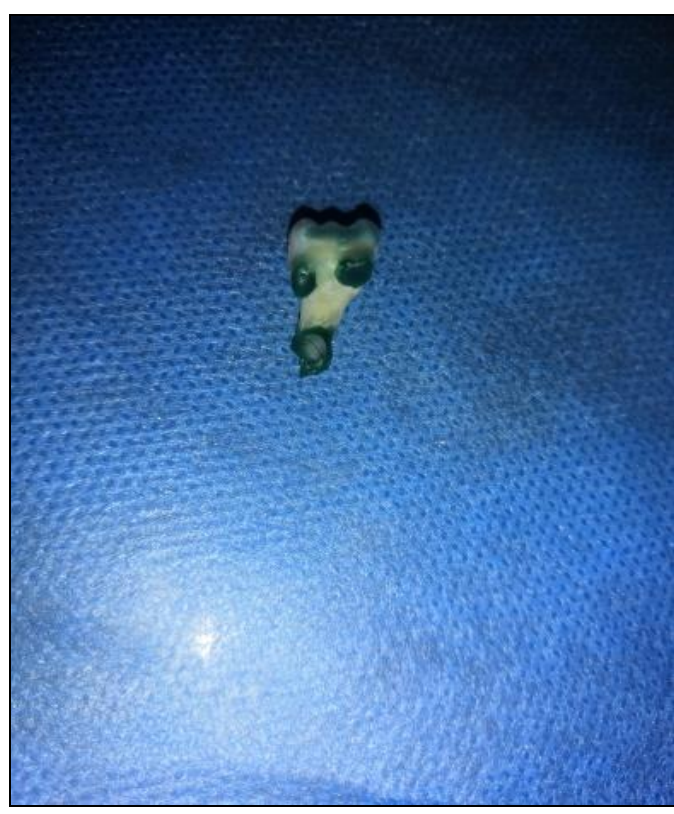

Fig 13: The process of blocking the tooth atom with adhesive wax

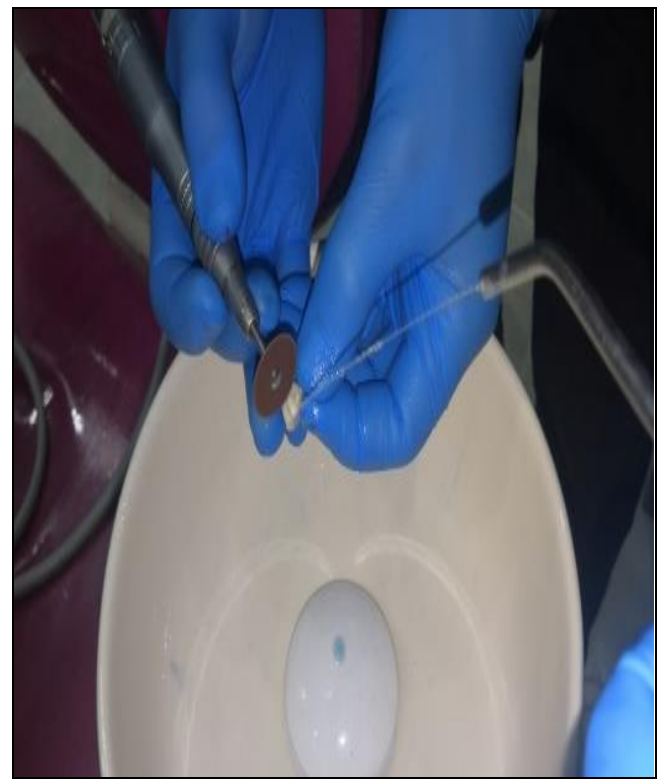

Fig 15: The process of cutting a tooth from the middle of the filling

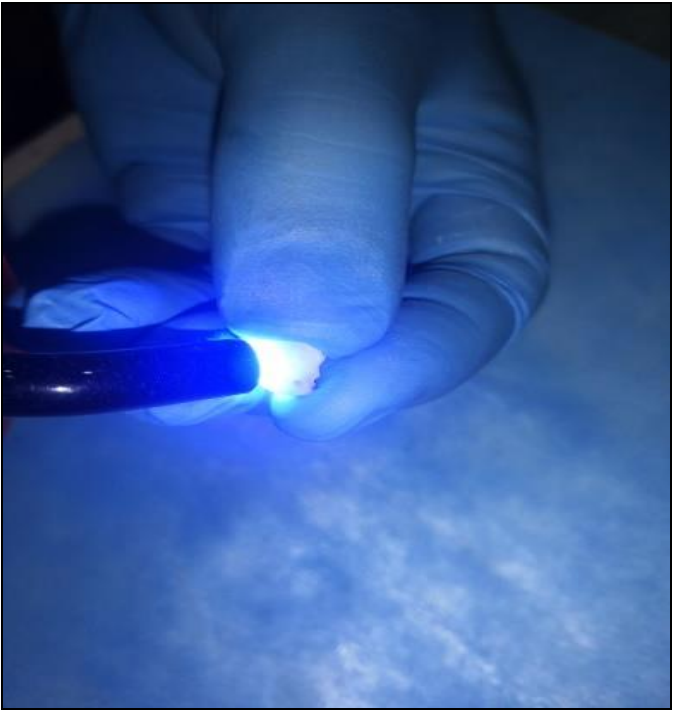

Fig 12: hardening of the composite

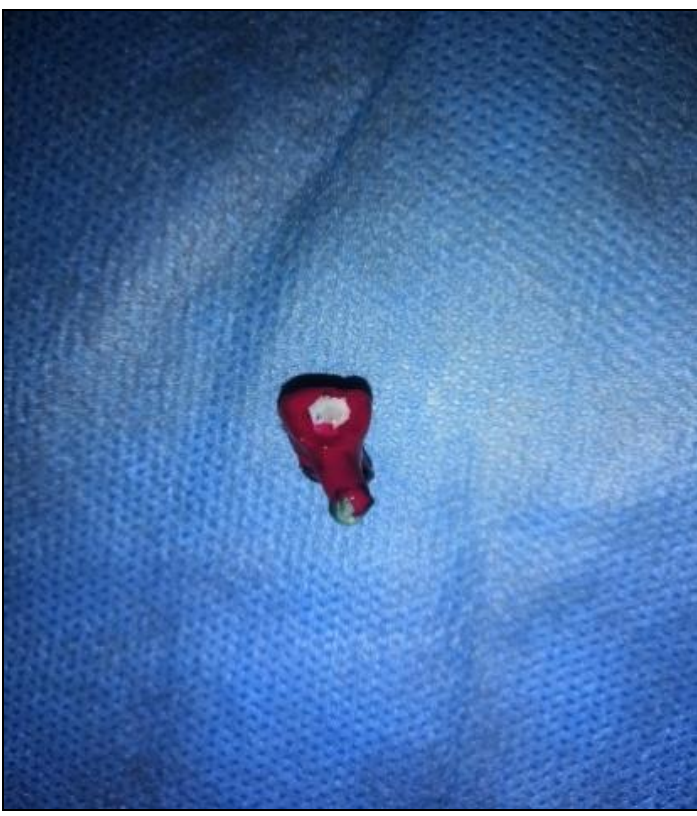

Fig 14: The process of covering the tooth with nail polish

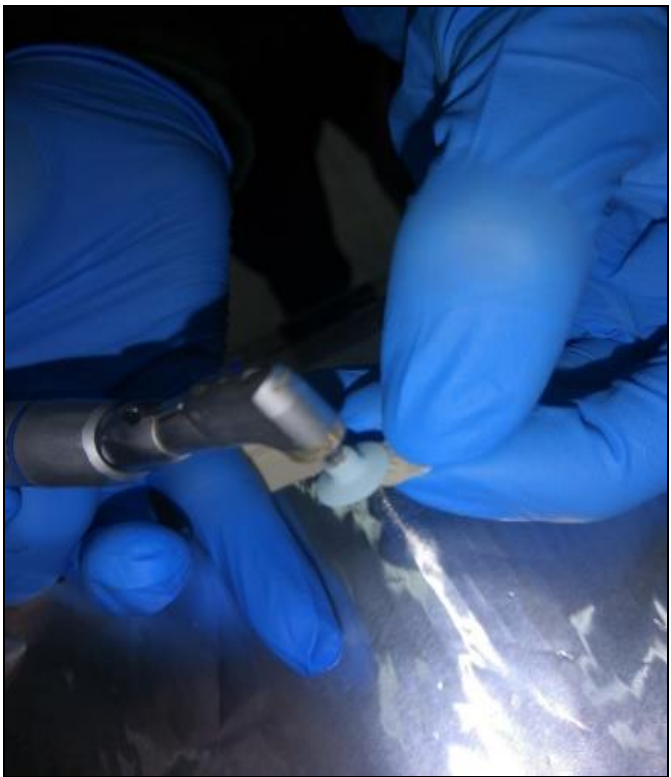

Fig 16: Smoothing the surface of the filling 


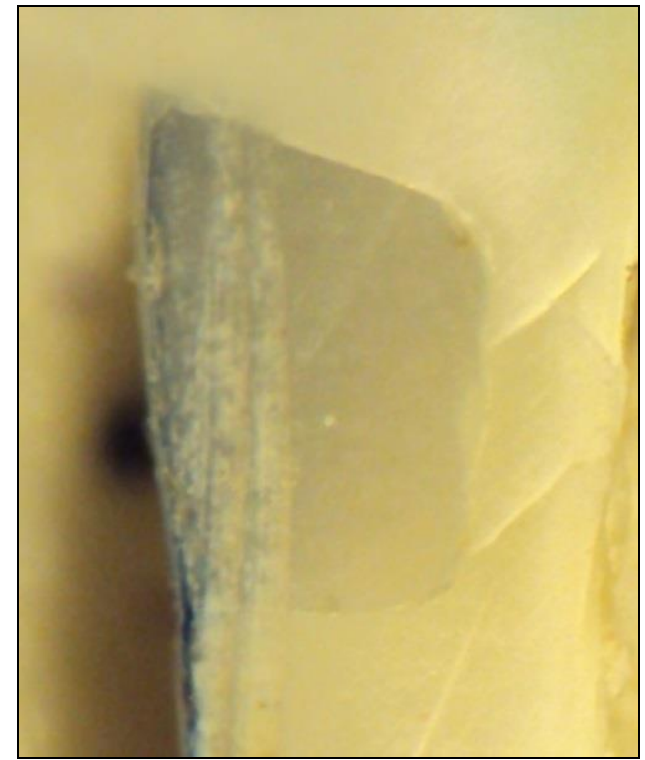

Fig 17: Degree No. (0) of the edge leakage.

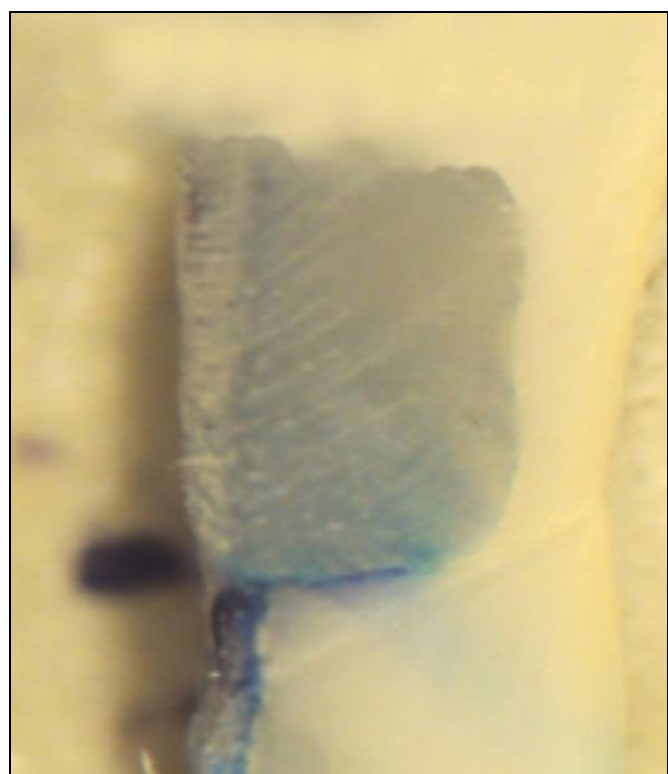

Fig 19: Degree No. 2 from the edge leakage.

\section{Results}

\section{Edge Leakage Study}

The degree of marginal leakage was monitored for each temporary molar of the temporal molar studied in the sample

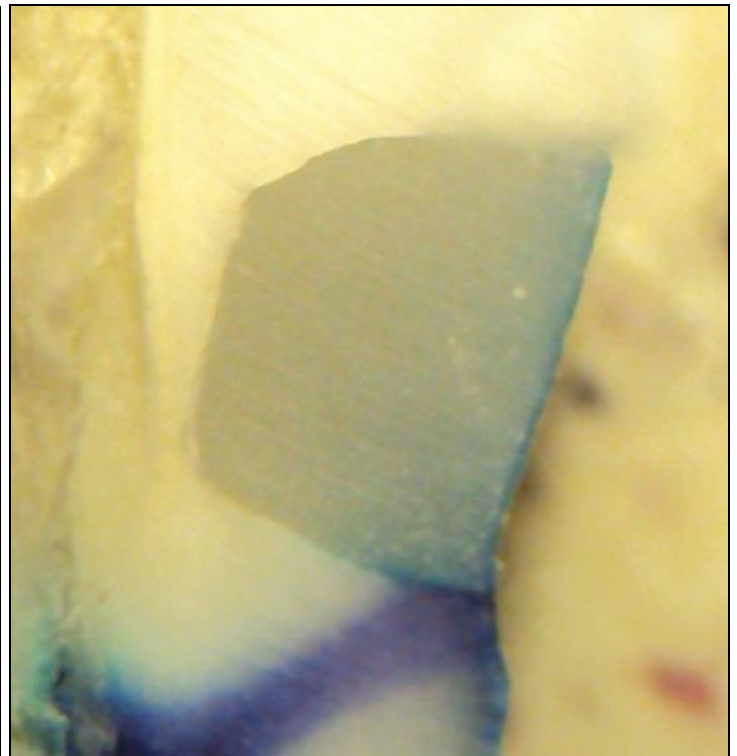

Fig 18: Degree No. 1 of the edge leakage

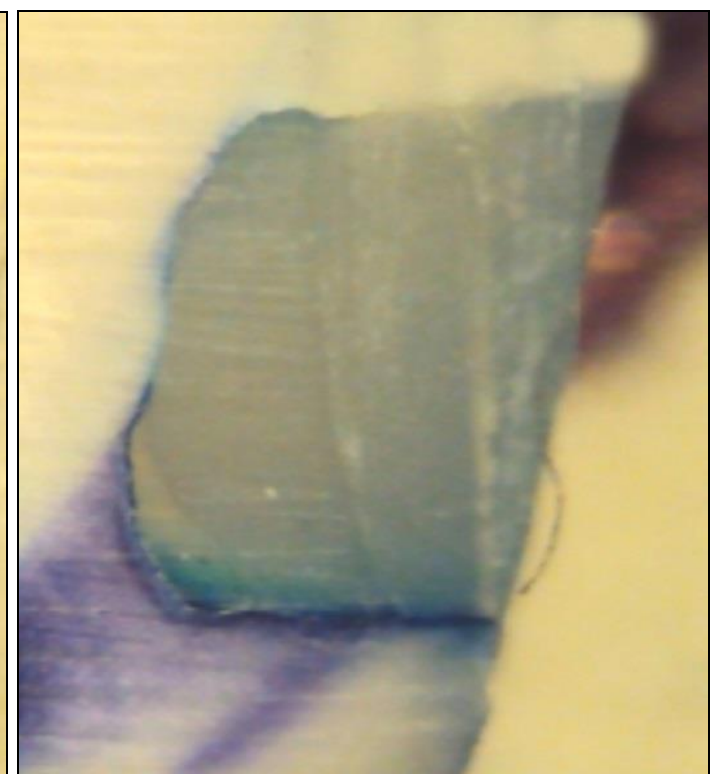

Fig 20: Degree No. 3 from the edge leakage

of the edge leakage, and each degree of edge leakage was given an incrementally increasing value according to the severity of the studied edge leakage as shown in the following table:

Table 2: Shows the approved scores for the edge leakage in the sample of the edge leakage and the corresponding value given for each grade.

\begin{tabular}{|c|c|}
\hline Edge Leakage Degree & Corresponding value given \\
\hline 0 & No edge leakage \\
\hline 1 & Edge seepage not exceeding half of padding \\
\hline 2 & My seepage leakage exceeded half of the filling and did not completely exceed \\
\hline 3 & Leakage of edges exceeding the ends of the filling \\
\hline
\end{tabular}

Then the effect of the restoration method followed on the frequency of the edge leakage pattern was studied in the sample of the edge leakage study. The results of the analysis were as follows:
Results of monitoring the degree of edge leakage in the sample of edge leakage according to the method of restoration followed: 
Table 3: Shows the results of monitoring the degree of edge leakage in the sample of edge leakage according to the method of restoration followed.

\begin{tabular}{|c|c|c|c|c|c|c|c|c|c|c|}
\hline \multicolumn{5}{|c|}{ Percentage \% } & \multicolumn{5}{|c|}{ Number of temporary molars } & \multirow[b]{2}{*}{$\begin{array}{l}\text { The followed } \\
\text { method of } \\
\text { restoration }\end{array}$} \\
\hline Total & $\begin{array}{l}\text { Marginal } \\
\text { leakage } \\
\text { exceeded the } \\
\text { edges of the } \\
\text { filling }\end{array}$ & \begin{tabular}{|c|}
$\begin{array}{c}\text { Marginal } \\
\text { leakage } \\
\text { exceeded half } \\
\text { of the filling } \\
\text { but not all the } \\
\text { filling }\end{array}$ \\
\end{tabular} & $\begin{array}{l}\text { Marginal } \\
\text { leakage does } \\
\text { not exceed } \\
\text { half of the } \\
\text { filling }\end{array}$ & $\begin{array}{c}\text { There's no } \\
\text { marginal } \\
\text { leakage }\end{array}$ & Total & \begin{tabular}{|} 
Marginal \\
leakage \\
exceeded the \\
edges of the \\
filling
\end{tabular} & \begin{tabular}{|c|}
$\begin{array}{c}\text { Marginal } \\
\text { leakage } \\
\text { exceeded half } \\
\text { of the filling } \\
\text { but not all the } \\
\text { filling }\end{array}$ \\
\end{tabular} & \begin{tabular}{|c|} 
Marginal \\
leakage does \\
not exceed \\
half of the \\
filling \\
\end{tabular} & $\begin{array}{l}\text { There's no } \\
\text { marginal } \\
\text { leakage }\end{array}$ & \\
\hline 100 & 0 & 5.0 & 50.0 & 45.0 & 20 & 0 & t & 10 & 9 & \begin{tabular}{|c|} 
Restoration of \\
temporary molar \\
with application of \\
$2 \%$ chlorohydroxine \\
solution
\end{tabular} \\
\hline 100 & 0 & 30.0 & 50.0 & 20.0 & 20 & 0 & 6 & 10 & 4 & \begin{tabular}{|c|} 
Restoration of \\
temporary molar \\
without application \\
of $2 \%$ \\
chlorohydroxine \\
solution \\
\end{tabular} \\
\hline
\end{tabular}

The percentage of the results of monitering the degree of marginal leakage in a sample of studying of the marginal leakage according to the followed method of restoration.

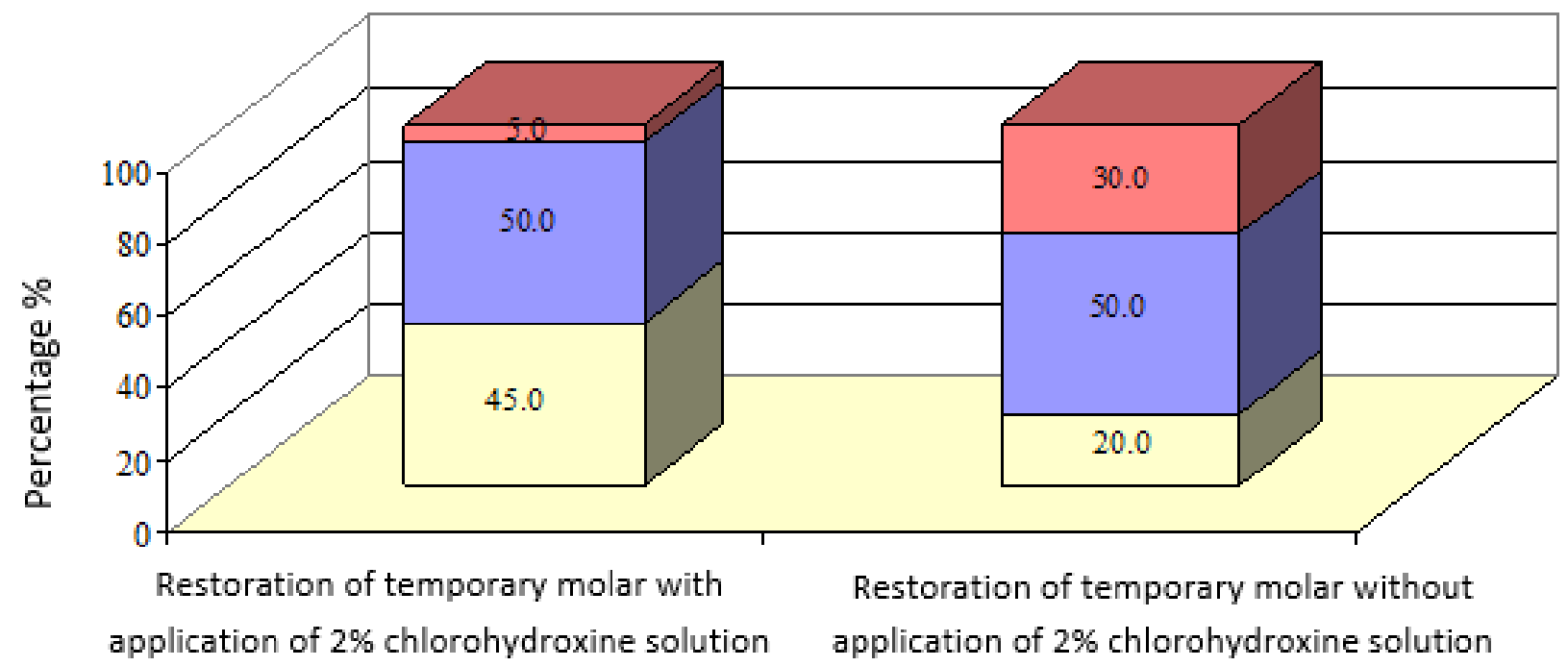

The followed method of restoration

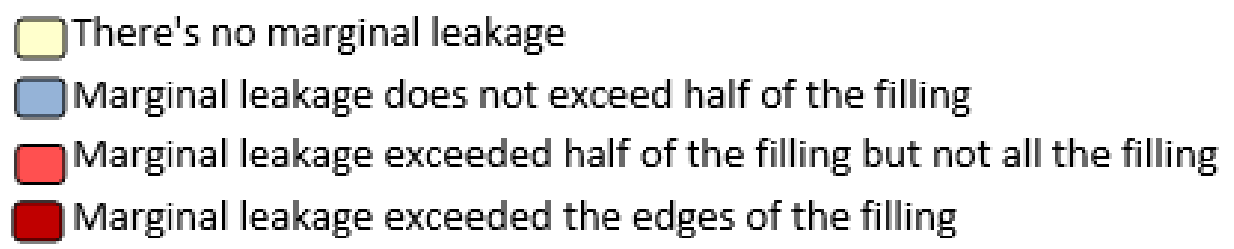

Scheme 1: Represents the percentage of the edge leakage in the sample of the edge leakage according to the method of restoration followed.

Studying the effect of the restoration method used on the degree of edge leakage :

Mann-Whitney U test was performed to study the significance of differences in the frequency of the edge leakage between the restoration group with applying $2 \%$ chlorhexidine solution and the restoration group without applying the $2 \%$ chlorhexidine solution in the sample of the edge leakage study as follows:

\section{Rank Statistics}

Table 4: shows the average rank of the degree of edge leakage in the sample of edge leakage according to the restoration method.

\begin{tabular}{|c|c|c|}
\hline \multicolumn{3}{|c|}{ Studied variable = degree of marginal leakage } \\
\hline The method of restoration followed & the number of temporary moral periods & of average rank \\
\hline Restoration of temporarily millet with application of chlorhexidine solution 2\% & 20 & 16.75 \\
\hline Restoration of the temporarily molar without applying chlorhexidine solution $2 \%$ & 20 & 24.25 \\
\hline
\end{tabular}




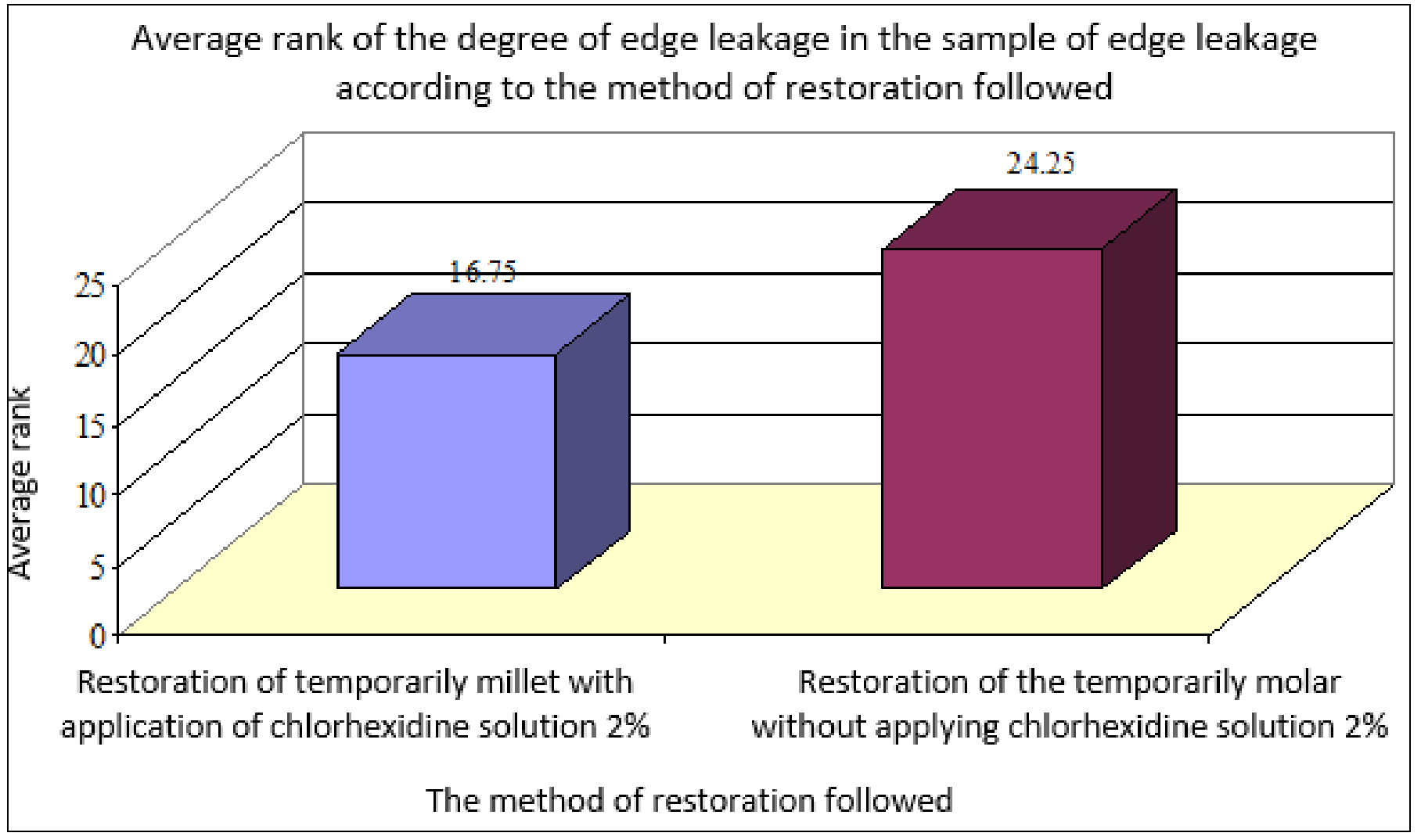

Scheme 2: Represents the average rank of the degree of edge leakage in the sample of edge leakage according to the method of restoration followed.

\section{Mann-Whitney U test results}

Table 5: shows the results of the Mann-Whitney U test to study the significance of the differences in the degree of edema leakage between the restoration group with applying the solution of chlorhexidine $2 \%$ and the restoration group without applying the solution of chlorhexidine $2 \%$ in the sample of the edging leakage.

\begin{tabular}{|c|c|c|}
\hline \multicolumn{3}{|c|}{ Studied variable = degree of marginal leakage } \\
\hline The value of U for Mann-Whitney & is the value of the significance level & indicative of differences \\
\hline 125.0 & 0.026 & There are significant differences \\
\hline
\end{tabular}

The above table shows that the value of the significance level is smaller than the value 0.05 , that is, at the $95 \%$ confidence level there are statistically significant differences in the frequency of the edge leakage between the restoration group with the application of chlorhexidine solution $2 \%$ and the restoration group without applying the solution of chlorhexidine $2 \%$ in the sample of the edge leakage study By examining the mean mean values, we conclude that the degree of marginal leakage in the restoration group with the application of chlorhexidine solution $2 \%$ was lower than that in the restoration group without applying the chlorhexidine solution $2 \%$ in the sample of the edge leakage study.

\section{Discussion \\ Discuss the results of the edge leakage}

The sealing of the ivory canals, and the ability to stop the edge seepage are the most important factors in the success of the resin restorations ${ }^{[6]}$. Especially when it comes to having an active necrotic activity in the patient, poor oral care, and unhealthy eating habits ${ }^{[7]}$.

The results of studies conducted on temporary teeth using chlorhexidine solution and in various methods (such as studying the forces of association and the edge leakage) have shown conflicting results in terms of impact.

However, none of those studies related to restoration techniques using resin composites in etching (stratigraphy - adjacent) in the temporary molars mentioned in the medical literature were not able to achieve the level (0) of the degree of edge leakage in most of the results ${ }^{[8]}$.

As the solidification of the compound resin often results in a decrease in the volume of the material compared to its size before photosynthetic hardening, which is known as polimerization contraction.

The resinous materials are subjected to edge seepage due to their properties, as they suffer from the occurrence of polimerization contraction when conducting photosynthesis, and because the coefficient of thermal expansion coefficient differs from the dental structures applied to them, the absorption of water and oral fluids when exposed to the oral medium, and the sensitivity of the application technique to pollution. Humid ${ }^{[9]}$.

There are many techniques that were used to measure the edge leakage, including the compressed air technology, the use of a method that relies on bacterial leakage, or the use of radioactive signs in the measurement of edge leaks markers radioactive, or the use of electrochemical investigation of the edge leakage electrochemical investigations, or the use of scanning electron microscopy scanning electron microscopy, in addition to using dye penetration technology ${ }^{[10]}$.

The pigmentation method is used to measure the edge leakage as a fast, easy and inexpensive technique that does not need many tools and its results are good (Camps and Pashley 
2003). Measuring the amount of pigment leakage in the performed sections is the most commonly used edge leakage measurement technique ${ }^{[11]}$.

A blue pigment with a concentration of $1 \%$ was used, as it is the most widely used marker in edge leakage studies that depend on pigmentation ${ }^{[12,13]}$.

Sridhar, Moses et al. 2016) where the depth of pigment insertion in the interface between the age and the resinous substance expresses the degree of edge seepage, and the closer the pigment is to the depth of the hole, the greater the edge of the seepage.

The results of the current study showed a slight improvement in reducing the marginal leakage when applying the solution of chlorhexidine $2 \%$ to the temporary dentine compared to the group in which chlorhexidine was not applied.

The results of our study were consistent with a study (Darabi and Eftekhari 2009) and found that the application of chlorhexidine solution $2 \%$ after acidic scratching with - or without additional washing of chlorhexidine residues - and before applying the excite binding substance had no negative effect, as it did not increase the values of the edge leakage at the edge Enamel and ivory pits for class 5 restoration with tetric ceram,

\section{Conclusions and recommendations}

Within the limits of this study and within the results obtained, we can conclude the following:

1. That the application of chlorhexidine solution $2 \%$ after acidic scratching improved the process of association with temporary dentine.

2. The application of $2 \%$ chlorhexidine solution did not adversely affect the marginal leakage of the resin material after acid scratching.

3. The inability of the application of chlorhexidine solution $2 \%$ to reduce the edge leakage

4. We recommend applying the $2 \%$ chlorhexidine solution to temporary dentine because of its positive effect in improving the binding process.

5. It is preferred for the practitioner to apply the chlorhexidine solution $2 \%$ after the acid scratching procedure.

6. It is preferred to expand the study to include the difference in the application time of $2 \%$ chlorhexidine solution on the temporary teeth.

7. It is preferred to expand the study to include the application of $0.5 \%$ chlorhexidine solution and indicate the extent of its effect on the temporary teeth.

\section{References}

1. Bansal S, Pandit IK, Srivastava N, Gugnani N. echniquesensitivity of dentin-bonding agent application: the effect on shear bond strength using one-step self-etch adhesive in primary molars: an in vitro study. Journal of Indian Society of Pedodontics and Preventive Dentistry. 2010; 28(3): 183 .

2. Bengtson CRG, Bengtson AL, Bengtson NG, Turbino, ML. Do the origins of primary teeth affect the bond strength of a self-etching adhesive system to dentin?. Brazilian oral research. 2010; 24(3):355-360.

3. Blackwood JA, DC Dilley et al. Evaluation of pumice, fissure enameloplasty and air abrasion on sealant microleakage." Pediatric dentistry. 2002; 24(3):199-203.

4. Camps J, D Pashley. Reliability of the dye penetration studies." Journal of Endodontics. 2003; 29(9):592-594.

5. Casagrande L, Brayner R, Barata JS, De Araujo FB.
Cervical microleakage in composite restorations of primary teeth-in vitro study. Journal of dentistry. 2005; 33(8):627-632.

6. Darabi F, M Eftekhari. "Effect of Chlorhexidine on Microleakage of Composite." Journal of Dentistry of Tehran University of Medical Sciences. 2009; 6(1):1622.

7. Hickel R, Manhart J. Longevity of restorations in posterior teeth and reasons for failure. Journal of Adhesive Dentistry. 2001; 3(1).

8. Mair L, Padipatvuthikul P. Variables related to materials and preparing for bond strength testing irrespective of the test protocol. Dental materials. 2010; 26(2):e17-e23.

9. Matis BA, Cochran MJ, Carlson TJ, Guba C, Eckert GJ. A three-year clinical evaluation of two dentin bonding agents. The Journal of the American Dental Association. 2004; 135(4):451-457.

10. Mithiborwala SH, Chaugule V, Munshi A, Patil V. Comparative Evaluation of the Adhesive Properties of Two Generations of Dentin Bonding Agents by Checking the Microleakage in the Primary Teeth: An in vitro Study. International journal of clinical pediatric dentistry. 2011; 4(3):195.

11. Nunes MCP, EB Franco et al. Marginal microleakage: critical analysis of methodology. Salusvita 24(3):487502.

12. Prabhakar A, SA Murthy et al. Comparative evaluation of the length of resin tags, viscosity and microleakage of pit and fissure sealants-an in vitro scanning electron microscope study. Contemporary clinical dentistry. 2011; 2(4):324.

13. Sridhar LP, J Moses et al. "omparative Evaluation of the Marginal Sealing Ability of two Commercially Available Pit and Fissure Sealants, 2016.

14. Stalin A. Comparative evaluation of tensile bond strength, fracture mode and microleakage of fifth, and sixth generation adhesive systems in primary dentition (Doctoral dissertation, Ragas Dental College \& Hospital, Chennai), 2005.

15. Yaseen SM, Reddy VS. Comparative evaluation of shear bond strength of two self-etching adhesives (sixth and seventh generation) on dentin of primary and permanent teeth: An in vitro study. Journal of Indian Society of Pedodontics and Preventive Dentistry. 2009; 27(1):33. 